\title{
Modeling human-bed interaction: the predictive value of anthropometric models in choosing the correct bed support
}

\author{
Vincent Verhaert ${ }^{\mathrm{a},}{ }^{,}$, Hans Druyts ${ }^{\mathrm{b}}$, Dorien Van Deun ${ }^{\mathrm{a}}$, Tom De Wilde ${ }^{\mathrm{b}}$, Karel Van Brussel ${ }^{\mathrm{b}}$, Bart Haex ${ }^{\mathrm{a}}$ \\ and Jos Vander Sloten ${ }^{\mathrm{a}}$ \\ ${ }^{a}$ Biomechanics section, Mechanical Engineering Department, Katholieke Universiteit Leuven, Celestijnenlaan \\ 300c, bus 2419, 3001 Leuven, Belgium \\ ${ }^{\mathrm{b}} R \& D$ Custom8 NV, Ridderstraat 26, Leuven, Belgium
}

\begin{abstract}
The sleep system (i.e. the combination of mattress and bed base) is an important factor of the sleep environment since it allows physical recuperation during sleep by providing proper body support. However, various factors influence the interaction between the human body and the sleep system. Contributing factors include body dimensions, distribution of body weight and stiffness of the sleep system across the mattress surface. During the past decade, the rise of several new bedding technologies has made it increasingly difficult for the consumer to select a proper sleep system. Therefore, this study presents a method to model human-bed interaction in order to objectively predict the ideal sleep system for a particular individual. The proposed method combines a personalized anthropometric model with standardized load-deflection characteristics of mattress and bed base. Results for lateral sleep positions show a root mean square deviation of $11.9 \pm 6.1 \mathrm{~mm}$ between modeled spine shapes and validation shapes, derived from 3D surface scans of the back surface. The method showed to be a reliable tool to individually identify the sleep system providing superior support from a variety of possible mattress-bed base combinations.
\end{abstract}

Keywords: ergonomic design criteria, sleep system optimization, personalized body support, mechanical bed characteristics, bed support

\section{Introduction}

Over the past ten years, an increased effort has been taken to incorporate ergonomic aspects in the design of sleep systems (i.e. the combination of mattress and bed base). Whereas mattresses with a homogeneous stiffness distribution used to be the standard, nowadays most systems in Western countries consist of distinct comfort zones in order to properly contour the human body [7]. In addition, the importance of a personalized approach when purchasing a sleep system is being emphasized more and more (e.g. different body types need different sleep systems) [11, 25]. However, various technical innovations - with new materials being deployed in all components of the sleep system - have hampered a well-considered purchase of the consumer. Consequently, there is a strong demand for scientifically validated decision criteria to assign a sleep system to a specific person.

The most important function of sleep systems is to provide proper support in a way that allows the human body to recover from daily activities. Optimal recovery is achieved when the spine is in its natural, unloaded shape, allowing muscle relaxation and rehydration of the intervertebral discs $[16,14]$. In this context, optimizing body support refers to aligning the spine towards its shape during stance, yet with a somewhat flattened lumbar lordosis due to the changed working axis of gravity [4]. Since spinal alignment is the result of the complex biomechanical interaction of the human body and the sleep system, it is influenced by a variety of factors, including not only the mechanical characteristics of the bedding system (more specifically the stiffness distribution),

*Corresponding author. E-mail: vincent.verhaert@mech.kuleuven.be. 
but also the subject's weight distribution and body dimensions. Ideally, all these factors are considered when purchasing a new sleep system.

Several methods have been described in literature to assess bed comfort, such as spine shape reconstruction $[13,3,10]$, pressure mapping [21, 22, 15], electromyography (EMG) $[13,12]$ and subjective evaluations $[17,1]$. In this study, spinal alignment will be considered as the main criterion to assess bed comfort for the following reasons. First, spinal alignment provides a global measure of the deformation of the human body while sleeping [18]. Second, although pressure distribution is of primary concern for the prevention of pressure ulcers in bedridden patients [21], it is sufficient to avoid concentrated pressure peaks when considering a general (healthy) population. Third, at present the validity of EMG to assess body support on sleep systems is rather poorly documented and remains subject to discussion [13].

This study presents a method to model human-bed interaction in order to objectively predict the ideal mattress-bed base combination for a particular person. The method combines a personalized anthropometric model of the sleeper with information on the mechanical behavior of mattress and bed base. Since it is based on standardized mechanical characteristics (load-deflection properties), it is independent of bedding technology. Consequently, the presented method enables to individually identify the sleep system with superior support properties from a range of distinct mattress-bed base combinations.

\section{Methodology}

\subsection{Participants}

Eighteen subjects (nine males, nine females; aged $28.5 \pm 4.7$ years) participated in the study. Anthropometric information was determined by means of silhouette extraction in both the sagittal and frontal plane (Ikélo, Custom8, Leuven, Belgium).

\subsection{Bedding systems}

Three types of bed bases were included: 1) a homogeneous box-spring, 2) a multi-zone slatted base, and 3) a multi-zone mesh base. They were combined with three standard types of mattresses: 1) a multizone pocket spring mattress 2) a multi-zone latex mattress, and 3) a homogeneous polyurethane foam mattress. Hence, a total of nine mattress-bed base combinations were accounted for.

\subsection{Simulation}

\subsubsection{Human body shape model}

A generic surface model of the human body was used that consists of consecutive superellipses representing the transverse cross sections of the human body. Personalization is achieved by optimizing the semi-diameters and ellipse order to match the measured body contours (Figure 1). Prior research [24] fully described this procedure and validated the resulting models with 3-D surface scans of the trunk, showing a mean unsigned distance of $9.77 \mathrm{~mm}$ between modeled and scanned surface meshes.

The surface model is further extended by integrating a distribution of body weight over the different body parts. Appropriate slice masses were determined based on density values of human body segments described in literature [6]. The volume-based slice masses were scaled in such way that their sum corresponded to the measured body weight - reduced with the estimated weight of head and feet, which were not modeled.

\subsubsection{Characterization of sleep system components}

Load-deflection curves were determined for each bed base and each mattress separately using a flat and circular stamp (diameter $210 \mathrm{~mm}$, rounded with a 5 $\mathrm{mm}$ radius at the lower edge). Travel speed of the

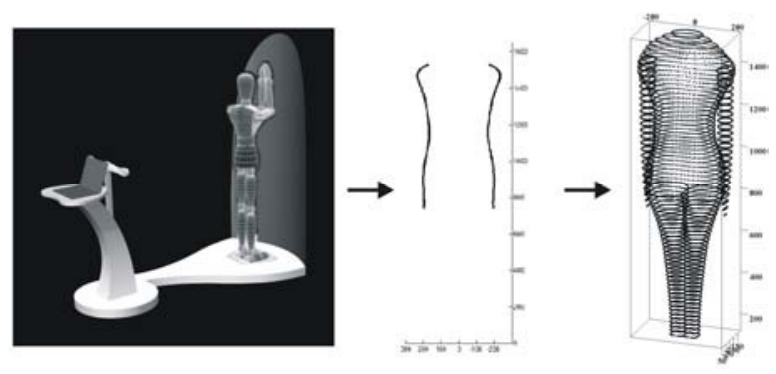

Figure 1: Model personalization based on measured anthropometrics 

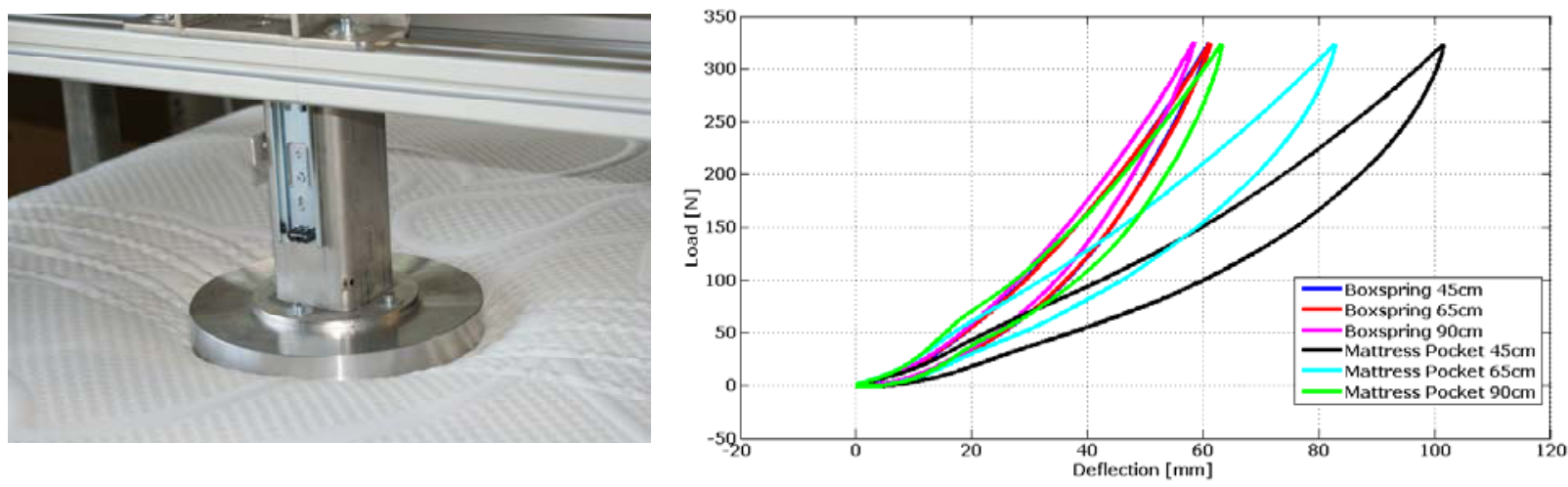

Figure 2: Measurement set-up and sample load-deflection characteristics for three locations on a box-spring base and pocket spring mattress

stamp was kept constant at $120 \mathrm{~mm} / \mathrm{min}$. Five discrete locations were characterized along the center line of the sleep systems at respectively $45 \mathrm{~cm}, 65 \mathrm{~cm}$, $75 \mathrm{~cm}, 90 \mathrm{~cm}$ and $100 \mathrm{~cm}$ of the head end. Loadrelief cycles were repeated at least three times and approximated by polylines. Only the third cycle was retained for further calculations. Figure 2 illustrates the measurement set-up and provides some examples of load-deflection characteristics for three locations on a box-spring base and a pocket spring mattress.

\subsubsection{Simulation of spine shape}

In order to simulate human-bed interaction based on the personalized human model and the loaddeflection characteristics several considerations are taken into account.

First of all, the combined mechanical behavior of mattress and bed base is simulated based on the separate load deflection characteristics. On that account, the local stiffness ratio of mattress and bed base has shown to be a predicting factor to determine the equivalent force that is exerted by the mattress on the bed base when the complete sleep system is loaded by a specific force.

A second consideration involves the distinct contact surface (both area and shape) of the human body segments with respect to the circular stamp that was used to determine the load-deflection characteristics. Therefore two correction factors were defined as follows:

$$
\begin{aligned}
C_{1} & =\frac{A_{r e f}}{A_{e f f}} \\
C_{2} & =\frac{a}{h}
\end{aligned}
$$

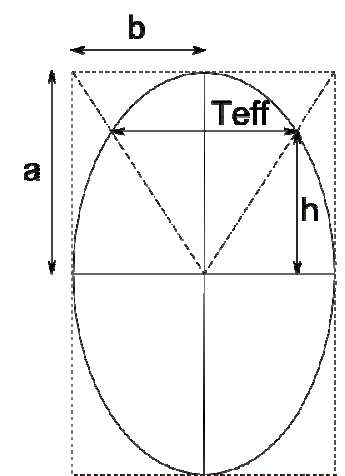

Figure 3: Factors that determine the correction factors to account for the variable shape and area of the contact surface with respect to the circular stamp.

with $A_{\text {ref }}$ the contact area of the indentation stamp, $A_{\text {eff }}$ the effective contact area of the body segment, $a$ the lateral semi-diameter of the superellipse and $h$ the distance between the effective contact line $\left(T_{\text {eff }}\right)$ and the center of the superellipse. Figure 3 clarifies these factors. The enclosing rectangle of the superellipse defines the effective contact line of the cross section with the mattress surface $\left(T_{e f f}\right)$. Numerical integration over subsequent superelliptic cross sections determines the effective contact area $A_{\text {eff. }}$. Both correction factors were applied to the weights of the corresponding body segments.

The human model was segmented at 20,30, 45 and $55 \mathrm{~cm}$ below the shoulder joint to assure that the centers of the body segments coincided with the locations for which indentation characteristics were determined (with the shoulder joint coinciding with the location at $45 \mathrm{~cm}$ of the head end). Each segment consisted of five consecutive superellipses corres- 


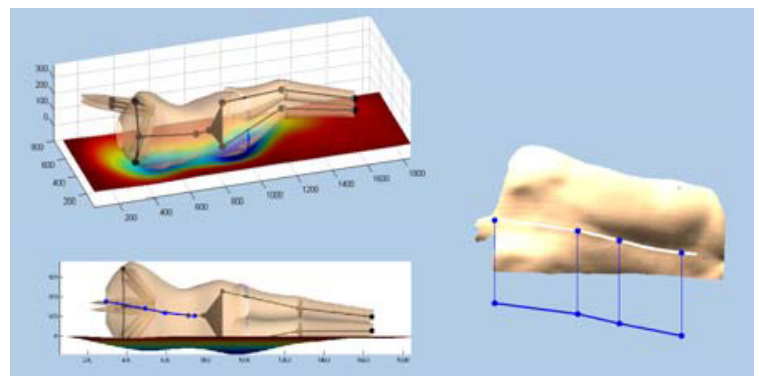

Figure 4: Simulation (left) and validation (right) of a particular mattress-bed base combinations for one of the participants.

ponding to a segment length of $10 \mathrm{~cm}$. Based on the corrected weights of the body segments their displacement was calculated. Finally, the line through the centers of the body segments represented the simulated shape of the spine.

\subsection{Validation}

\subsubsection{Measurements}

Validation of the predicted spine shape was provided by a 3-D surface scan of the back surface (zSnapper, Vialux, Chemnitz, Germany). Based on surface curvature information the locations of the following landmarks were determined: the vertebra prominens, the dimples of the posterior superior iliac spine and the sacrum point [5]. The line through the spinous processes was reconstructed by means of an active contour model iterating on a weighted combination of surface curvature information and lateral asymmetry information [8]. Prior research validated this approach with full spine CT measurements, reporting a root mean square deviation of $2.6 \mathrm{~mm}$ in lateral sleep positions [9].

In order to compare the validation shapes with the simulated spine shapes, they were subsampled to discrete points corresponding to the locations of the body segments at $20 \mathrm{~cm}, 30 \mathrm{~cm}$ and $45 \mathrm{~cm}$ below the shoulder point. Figure 4 demonstrates the simulation and validation of a particular mattress-bed base combination for one of the participants.

\subsubsection{Analysis}

An ergonomic bed score for lateral sleep postures (EBS L) was defined based on the weighted combination of the angle of the least square line through the spine points $(\alpha)$ and the angle between the lumbar and the thoracic part of the spine $(\gamma)$ :

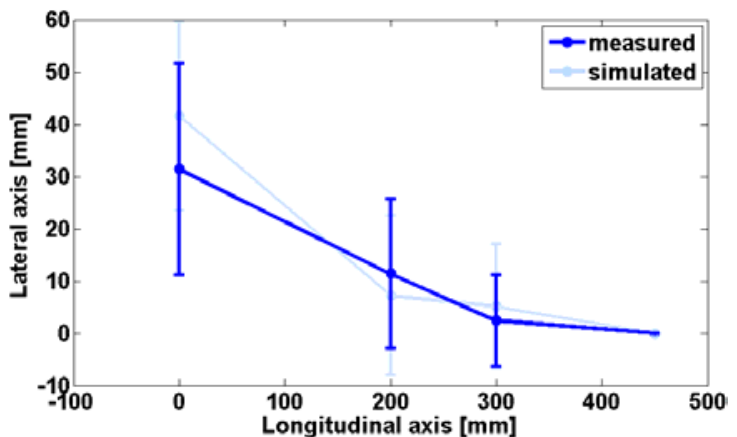

Figure 5: Simulated and measured spine shape averaged over all mattress-bed base combinations of the entire subject population. The zero point on the longitudinal axis corresponds to the shoulder point.

$$
E B S_{-} L=10 \cdot\left(1-\exp \left(\frac{-0.4}{5 \cdot(|\alpha|+0.25 \cdot|\gamma|)}\right)\right)
$$

This score (between 0 and 10) accounts for both the orientation of the spine with respect to the horizontal plane and for the deviation of the spine from a straight line. Based on the ESB L, a ranking of the tested mattress-bed base combinations was determined for each person separately.

\section{Results}

Table 1 gives an overview of the anthropometric data that was gathered to personalize the models along with the calculated weights of the different parts of the trunk. Figure 5 shows the average simulated and measured spine shapes along with the corresponding variation whereas table 2 presents the descriptive statistics of the ergonomic bed scores derived from the predicted and validated spine shapes. Both figure 5 and table 2 illustrate the validity of the presented method to predict quality of bed support in general. A more detailed, quantitative comparison of predicted spine shapes with the validation shapes revealed a root mean square deviation of $11.9 \pm 6.1$ $\mathrm{mm}$. In addition, it can be inferred from figure 6 that no effect of bed base type nor mattress type was noted on the accuracy of spine shape prediction $(\mathrm{p}=$ $0.22)$.

Finally, the rankings based on the measured shapes showed an overall correspondence of $85 \%$ with the rankings based on the simulated shapes. More specifically, the method correctly identified for 
Table 1

Summary statistics of measured anthropometric data for the entire subject population $(n=15)$

\begin{tabular}{lc}
\hline & Mean \pm std \\
\hline Body length [mm] & $1749 \pm 67$ \\
Body weight [kg] & $71.0 \pm 8.6$ \\
Shoulder height [mm] & $1410 \pm 57$ \\
Breast height [mm] & $1307 \pm 49$ \\
Waist height [mm] & $1112 \pm 44$ \\
Pelvis height [mm] & $921 \pm 38$ \\
Hip height [mm] & $847 \pm 42$ \\
Shoulder breadth [mm] & $440 \pm 29$ \\
Breast breadth [mm] & $376 \pm 31$ \\
Waist breadth [mm] & $342 \pm 14$ \\
Pelvis breadth [mm] & $392 \pm 21$ \\
Hip breadth [mm] & $405 \pm 22$ \\
Shoulder weight [kg] & $12.1 \pm 1.6$ \\
Breast weight [kg] & $10.3 \pm 1.3$ \\
Waist weight [kg] & $9.4 \pm 1.1$ \\
Pelvis weight [kg] & $18.3 \pm 2.2$ \\
\hline
\end{tabular}

16 out of 18 subjects the sleep system offering superior support. The two cases in which the best combination was not predicted, showed a very small difference in ESB L with the second best combination ( 0.29 and 0.25 respectively), indicating that the two superior combinations provided similar support.

\section{Discussion}

This study shows that it is feasible to individually assess quality of support provided by a particular mattress-bed base combination. During the past decade the importance of a personalized approach when choosing a sleep system has increasingly been emphasized. However, no clear decision criteria have been proposed to objectively quantify the effect of different sleep system components (e.g. mattress and bed base) on quality of body support. One of the underlying reasons for this lack of objective criteria is the existence of various influencing factors that determine the complex interaction between the human body and the sleep system. Consequently, this study aimed at providing a method to individually determine spine shape when lying in a lateral position on a particular sleep system. Its main asset is that it is independent on the type of mattress or bed base, making it suitable for decision support across brand specific technologies.

The presented method is based on the working hypothesis that body support is determined by individual body features on the one hand (e.g. body contours, weight distribution,...) and the mechanical behavior of the sleep system on the other hand. The former is accounted for by means of a personalized human model [24], whereas the latter is incorporated by

Table 2

Descriptive statistics of the predicted and validated ergonomic bed scores.

\begin{tabular}{lcc} 
& \multicolumn{2}{c}{ ergonomic bed scores. } \\
\hline & Simulation & Validation \\
\hline Mean & 5.20 & 6.09 \\
St. dev. & 1.09 & 1.83 \\
Max. & 8.07 & 9.99 \\
Min. & 3.70 & 3.37 \\
\hline
\end{tabular}

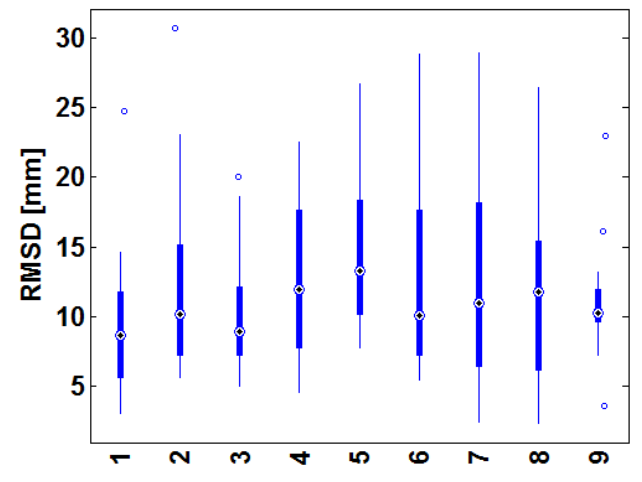

Mattress-bed base combination

Figure 6: Boxplots of the root mean square deviations (RMSD) between predicted and measured spine shapes for the nine possible mattress-bed base combinations

standardized load-deflection characteristics of mattress and bed base. Two correction factors were applied to account for differences in shape and contact area of distinct body segments. The resulting shapes were validated by means of spine shape reconstruction based on back surface measurements, showing an average root mean square deviation of $11.9 \mathrm{~mm}$. Furthermore, the distinct mattress-bed base combinations were ranked according to their ergonomic bed score for lateral sleep positions (ESB_L). Rankings based on the predicted spine shapes showed a good correspondence $(85 \%)$ with rankings based on the validated spine shapes. Moreover, the two persons for which the superior combination was not correctly identified showed very little difference in ESB L between the top two combinations.

Although the developed methodology provides an important first step towards objective decision criteria for sleep system selection, some limitations remain present. First, only lateral sleep positions were considered. In fact, healthy sleep is characterized by the presence of multiple position shifts throughout the night [2]. Therefore, it makes sense to incorporate 
spine shape assessment in supine and prone positions as well. A second limitation involves that body support is not the only factor that determines sleep comfort. Several high quality studies have shown that the thermal environment might affect sleep initiation and sleep depth $[19,20]$. Since the bed microclimate is highly affected by the thermal properties of the sleep system, information on thermal insulation and vapor permeability should be considered as well when choosing a sleep system. Therefore, future work will look at characterizing the thermal interactions between the human body and the sleep system that take place during sleep [23].

In conclusion, the presented work provides a first step towards the development of objective criteria to evaluate support properties of sleep systems on a personalized level. It was shown that spine shape could be predicted by combining a personalized anthropometric model with load-deflection behavior of mattress and bed base.

\section{References}

[1] P. Buckle and A. Fernandes, Mattress evaluation - assessment of contact pressure, comfort and discomfort, Applied Ergonomics 29 (1998), 35-39.

[2] J. De Koninck, D. Lorrain and P. Gagnon, Sleep positions and position shifts in five age groups: an ontogenetic picture, Sleep 15 (1992), 143-149.

[3] J. W. DeVocht, D. G. Wilder, E. R. Bandstra and K. F. Spratt, Biomechanical evaluation of four different mattresses, Applied Ergonomics 37 (2006), 297-304

[4] P. Dolan, M. A. Adams and W. C. Hutton, Commonly adopted postures and their effect on the lumbar spine, Spine 13 (1988), 197-201.

[5] B. Drerup and E. Hierholzer, Automatic localization of anatomical landmarks on the back surface and construction of a body-fixed coordinate system, Journal of Biomechanics 20 (1987), 961-970.

[6] R. Dumas, L. Chèze and J.-P. Verriest, Adjustments to McConville et al. and Young et al. body segment inertial parameters, Journal of Biomechanics 40 (2007), 543-553.

[7] B. Haex, Back and bed: ergonomic aspects of sleeping, CRC Press, Boca Raton, 2005.

[8] E. Hierholzer, Analysis of left-right asymmetry of the back shape of scoliotic patients, in Biostereometrics '85: Proc. SPIE 602, 1986, 266-271.

[9] T. Huysmans, Spine modeling from back shape data: an active contour and active shape model approach, Ph.D. Dissertation, Katholieke Universiteit Leuven, 2004.
[10]T. Huysmans, B. Haex, T. De Wilde, R. Van Audekercke, J. Vander Sloten and G. Van der Perre, A 3D active shape model for the evaluation of the alignment of the spine during sleeping, Gait \& Posture 24 (2006), 54-61.

[11]B. H. Jacobson, A. Boolani, G. Dunklee, A. Shepardson and H. Acharya, Effect of prescribed sleep surfaces on back pain and sleep quality in patients diagnosed with low back and shoulder pain, Applied Ergonomics 42 (2010), 91-97.

[12] J.-Y. Kim, S.-N. Min, M.-H. Lee, J.-H. Jeong, J.-H. An and Y.-S. Shin, Measurement of user experience to select a comfortable mattress, in: Design, User Experience, and Usability. Theory, Methods, Tools and Practice, A. Marcus, ed., Springer-Verlag, Berlin/Heidelberg, 2011, 449-458.

[13] R. Lahm and P. A. Iaizzo, Physiological responses during rest on a sleep system at varied degrees of firmness in a normal population, Ergonomics 45 (2002), 798-815.

[14]A. D. LeBlanc, H. J. Evans, V. S. Schneider, R. E. Wendt and T. D. Hedrick, Changes in intervertebral disc cross-sectional area with bed rest and space flight, Spine 19 (1994), 812-817.

[15] W. V. McCall, N. Boggs and A. Letton, Changes in sleep and wake in response to different sleeping surfaces: a pilot study, Applied Ergonomics, in press.

[16]A. Nachemson and G. Elfström, Intravital dynamic pressure measurements in lumbar discs. A study of common movements, maneuvers and exercises, Scand J Rehabil Med Suppl 1 (1970), 1-40.

[17] S. J. Park, J. S. Kim and C.-B. Kim, Comfort evaluation and bed adjustment according to sleeping positions, Human Factors and Ergonomics in Manufacturing 19 (2009), 145-157.

[18]J. Ray, Spine configuration associated with various sleep surfaces, IEEE Engineering in Medicine and Biology Magazine 10 (1991), 33-36.

[19]R. Raymann, D. F. Swaab and E. Van Someren, Cutaneous warming promotes sleep onset, Am J Physiol Regul Integr Comp Physiol 288 (2005), R1589-R1597.

[20]R. Raymann, D. F. Swaab and E. Van Someren, Skin deep: enhanced sleep depth by cutaneous temperature manipulation, Brain 131 (2008), 500-513.

[21]S. Rithalia, Assessment of patient support surfaces: principles, practice and limitations, Journal of Medical Engineering \& Technology 29 (2005), 163-169.

[22]L. Tonetti, M. Martoni, M. Fabbri and V. Natale, Relationship between mattress technological features and sleep quality: an actigraphic study of healthy participants, Biological Rhythm Research 42 (2011), 247-254.

[23] V. Verhaert, G. De Bruyne, T. De Wilde, D. Van Deun, M. Vandekerckhove, D. Berckmans, J. Verbraecken, B. Haex and J. Vander Sloten, Bed temperature and humidity during sleep in mild thermal conditions, Sleep 34 (2011), A109.

[24] V. Verhaert, H. Druyts, D. Van Deun, D. Berckmans, J. Verbraecken, M. Vandekerckhove, B. Haex and J. Vander Sloten, The use of a generic human model to personalize bed design, in: Proc. $1^{\text {st }}$ Int Symp on Digital Human Modeling, Lyon, 2011, paper ID 2202.

[25] V. Verhaert, B. Haex, T. De Wilde, D. Berckmans, J. Verbraecken, E. De Valck and J. Vander Sloten, Ergonomics in bed design: the effect of spinal alignment on sleep parameters, Ergonomics 54 (2011), 169-178. 